\title{
ON REGULAR POINTS IN BURGERS TURBULENCE WITH STABLE NOISE INITIAL DATA
}

\author{
Christophe GIRAUD \\ Laboratoire de probabilités et modèles aléatoires, Université Pierre et Marie Curie, \\ et C.N.R.S. UMR 7599, 16, rue Clisson, 75013 Paris, France \\ Received 10 February 2000, revised 5 July 2001
}

ABSTRACT. - We study the set of regular points (i.e. the points which have not been involved into shocks up to time $t$ ) for the inviscid Burgers equation in dimension 1 when initial velocity is a stable Lévy noise. We prove first that when the noise is not completely asymmetric and has index $\alpha \in(1 / 2,1)$, the set of regular points is discrete a.s. and regenerative. Then, we show that in the case of the Cauchy noise, the set of regular points is uncountable, with Minkowsky dimension 0. ๑ 2002 Éditions scientifiques et médicales Elsevier SAS

AMS classification: 35Q53; 60H15; 60J65

Keywords: Burgers turbulence; Stable Lévy noise; Regular points

RÉSUMÉ. - Nous étudions l'ensemble des points réguliers (c'est-à-dire des points qui jusqu'au temps $t$ n'ont pas été impliqués dans des chocs) relatifs à l'équation unidimensionnelle de Burgers lorsque la vitesse initiale est un bruit stable de Lévy. Nous prouvons en premier lieu que pour un bruit non complètement asymétrique d'indice $\alpha \in(1 / 2,1)$, l'ensemble des points réguliers est discret p.s. et régénératif. Nous établissons par la suite que dans le cas du bruit de Cauchy l'ensemble des points réguliers est non dénombrable et de dimension de Minkowsky nulle. ๑ 2002 Éditions scientifiques et médicales Elsevier SAS

\section{Introduction}

Burgers equation

$$
\partial_{t} u+u \partial_{x} u=\mu \partial_{x x}^{2} u
$$

was first introduced as a simplification of the Navier-Stokes equation, by neglecting the terms of pressure and force (see [7]). This is the simplest PDE that captures the interaction of nonlinear wave propagation and viscosity. Burgers equation also appears in the study of growth interface such as ballistic aggregation and is proposed as a model to describe the formation of the superstructures of the universe, see [21] and references therein. 
There is a particular attention in the literature about the behavior of the Burgers turbulence when the viscosity $\mu \rightarrow 0$. It is known that the solution $u_{\mu}$ converges to $u_{0}=u$, where $u$ is the unique weak solution to the so-called inviscid Burgers equation

$$
\partial_{t} u+u \partial_{x} u=0
$$

satisfying the entropy condition. A physical interpretation of this solution is given by the model of sticky particles. At the initial time, infinitesimal particles are uniformly spread on the line, with initial velocity $u(., 0)$. They evolve with the dynamic of completely inelastic shocks. This means that the velocity of a particle only changes in case of collision, and when two (clusters of) particles collide, they stick and form a heavier cluster with conservation of masses and momenta.

There is an increasing interest in the inviscid Burgers equation (1) when the initial data $u(., 0)$ is a random process (see e.g. [7], [17] and [21]). Sinai [20] and Bertoin [4] have given a complete statistical description of the solution of (1) when $u(., 0)$ is a Brownian motion or a Lévy process with no positive jumps. Avellaneda and E [1,2], Ryan [18] and [9] have focused on the case of white noise initial velocity (i.e. when the initial velocity is the weak derivative of a Brownian motion). In fact, one could get a complete description of the system at a given time $t$ from the work of Groeneboom [12]. Bertoin [6] specified the shock structure of the solution of (1), when the initial velocity is a stable noise, i.e. when $u(., 0)$ is the weak derivative of a stable Lévy process of index $\alpha \in(1 / 2,2]$. Moreover, he has given large deviations estimates for the velocity (see [5]).

Regular points may be viewed as particles that have not been perturbed by the turbulence up to time $t$. She, Aurell and Frisch [19] as well as Janicki and Woyczynski [16] have investigated by numerical simulations the dimension of the set of regular points when the initial velocity is a fractional Brownian motion or a stable Lévy process. They have then conjectured that this dimension is related to the scaling exponent of these processes. Sinai [20] and Bertoin [4] have partially proved these results. In the same direction, Handa [13] has shown a link between the regularity of the initial data and the dimension of the set of regular points. In this paper we will turn our attention to the properties of the set of regular points (at a fixed time $t$ ) when the initial velocity $u(., 0)$ is a stable Lévy noise.

There are only two cases where the set $\mathcal{R}_{t}$ of regular points is not empty (see Bertoin [6]). The first is when the initial velocity is a non-completely asymmetric stable noise with index $\alpha \in(1 / 2,1)$. We will prove here that the set $\mathcal{R}_{t}$ of regular points is then discrete a.s. and follows a regenerative property. Moreover, between two consecutive regular points, clusters form an increasing sequence indexed by $\mathbb{Z}$. The second case where the set $\mathcal{R}_{t}$ is not empty is when the initial velocity is a Cauchy noise. We will check that $\mathcal{R}_{t}$ is then a.s. uncountable but has Minkowsky dimension 0.

In the second section, we recall some basic features and definitions on the inviscid Burgers equation and stable noises. The third section will be devoted to the case $\alpha \in(1 / 2,1)$, the next one to the Cauchy case, and we give in the last one numerical illustrations of both cases. 


\section{Preliminaries}

\subsection{Hopf-Cole solution of inviscid Burgers equation}

Assume that the initial potential $\psi_{0}$, defined by $\psi_{0}(x)-\psi_{0}(y)=\int_{x}^{y} u(z, 0) \mathrm{d} z$ (the integral is purely formal), has discontinuities only of the first kind and satisfies $\psi_{0}(z)=$ $\mathrm{o}\left(z^{2}\right)$, when $|z| \rightarrow \infty$. Then, when $\mu \rightarrow 0$, the unique solution of Burgers equation with viscosity $\mu>0$ converges (excepted on a countable set) to a weak solution of the inviscid Burgers equation, referred to as the Hopf-Cole solution, see [15], [8]. The rightcontinuous version of this solution is

$$
u(x, t)=\frac{x-a(x, t)}{t},
$$

where

$$
a(x, t)=\arg \max _{z}\left(\psi_{0}(z)-\frac{1}{2 t}(z-x)^{2}\right)
$$

is the (largest) location of the supremum of $z \rightarrow \psi_{0}(z)-\frac{1}{2 t}(z-x)^{2}$. One has the following geometrical interpretation: consider a realization of the initial potential $\psi_{0}$ and a parabola $z \mapsto(z-x)^{2} / 2 t+C$, where $C$ is chosen such that the parabola is strictly above the path of $\psi_{0}$ (Fig. 1). Let $C$ decrease until this parabola touches the graph of $\psi_{0}$. Then $a(x, t)$ is the largest abscissa of the contact points. One notices in particular that the solution is expressed in term of the initial potential, rather than the initial velocity.

The function $x \mapsto a(x, t)$ is non-decreasing and right continuous and its right continuous inverse $a \mapsto x(a, t)$ is known as the Lagrangian function, i.e. as the function that gives the position at time $t$ of the particle initially located in $a$.

A discontinuity of $x \rightarrow u(x, t)$ is called a shock and occurs when $x \rightarrow a(x, t)$ jumps, i.e. when $a(x, t) \neq a(x-, t)$, where $a(x-, t)$ refers to the left limit of $a(., t)$ in $x$. It happens when the function $\psi_{0}(z)-(z-x)^{2} / 2 t$ reaches its overall minimum at more than one location and then $a(x-, t)$ is the smallest of such locations. From the sticky particles point of view, the location of a shock corresponds to the location of a cluster

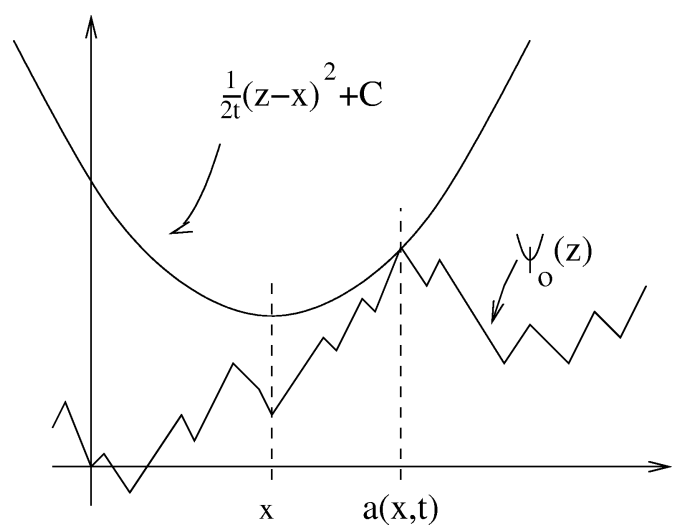

Fig. 1. Geometrical interpretation of $a(x, t)$. 


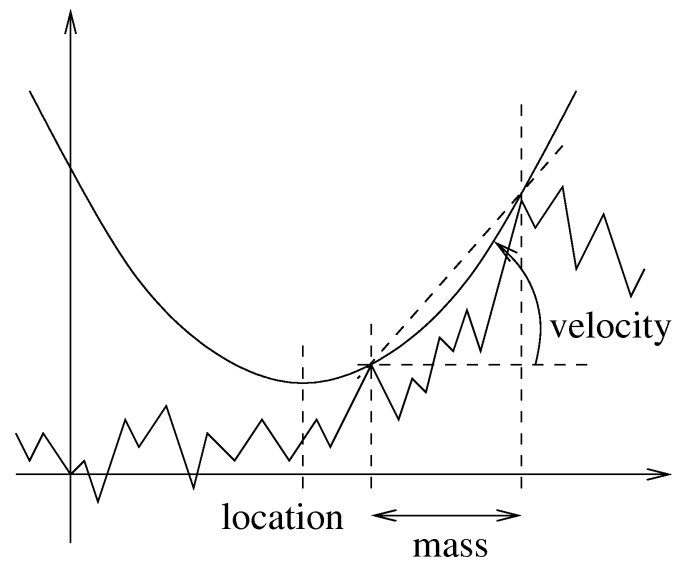

Fig. 2. Geometrical interpretation of a shock.

at time $t$. This cluster results from the aggregation of the particles initially located in $[a(x-, t), a(x+, t)]$; its velocity is (according to the conservation of masses and momenta)

$$
v(x)=-\frac{\psi_{0}(a(x))-\psi_{0}(a(x-))}{a(x)-a(x-)}=\frac{u(a(x))+u(a(x-))}{2} .
$$

The interval $[a(x-, t), a(x+, t)]$ is called a shock interval and $x$ a Eulerian shock point.

Finally, a Lagrangian regular point is a right and left accumulation point of the closed range of $a(., t)$. It represents the initial location of a particle that has not been involved into shocks up to time $t$. In the sequel, $\mathcal{R}_{t}$ will denote the set of Lagrangian regular points at time $t$.

\subsection{Stable Lévy noises}

Stable Lévy noises are the weak derivatives of stable Lévy processes. A stable Lévy process of index $\alpha \in(0,2]$ is a right continuous stochastic process with independent and stationary increments that fulfills the scaling property

$$
\psi_{0}(\lambda x) \sim \lambda^{1 / \alpha} \psi_{0}(x) \quad \forall \lambda>0,
$$

where $\sim$ means 'identity in law'. See Chapter VIII in [3] for much more on the topic. The requirement $\psi_{0}(z)=\mathrm{o}\left(z^{2}\right)$ when $|z| \rightarrow \infty$ imposes $\alpha>1 / 2$, so we will restrict our attention to this case.

First, let us recall some basic features on the two kind of processes to which we will turn our interest. A non-completely asymmetric stable Lévy process with index $\alpha \in(1 / 2,1)$ is a pure jump process with both positive and negative jumps. It has bounded variation a.s. A Cauchy process is the sum of a symmetric Cauchy process and a deterministic drift. Its index is $\alpha=1$. It has unbounded variation a.s. 
One deduces (see [19]) from the stationarity and the scaling property of $\psi_{0}$, that $u(., t)$ is a stationary process fulfilling the scaling property

$$
u(., t) \sim t^{(1-\alpha) /(2 \alpha-1)} u(., 1) .
$$

In particular, we can focus on time $t=1$ and we will write $a(x)=a(x, 1), u(x)=$ $u(x, 1)$ and $\mathcal{R}=\mathcal{R}_{1}$ in the sequel.

\section{The case $\alpha \in(1 / 2,1)$}

\subsection{Statement of the main results}

When $u(., 0)$ is a not completely asymmetric stable noise with index $\alpha \in(1 / 2,1)$, Bertoin [6] has proved that with probability one, there exists regular points. These are exceptional, in the sense that the set $\mathcal{R}$ of Lagrangian regular points has a.s. Lebesgue measure 0 .

Here, we shall first prove that regular points form a discrete set.

THEOREM 1. - Suppose that the initial potential $\psi_{0}$ is a stable Lévy process with index $\alpha \in(1 / 2,1)$ which is not completely asymmetric. Then a.s. the set $\mathcal{R}$ of Lagrangian regular points is discrete.

Let us denote thenceforth $\mathcal{R}=\left\{r_{n} ; n \in \mathbb{Z}\right\}$, where $r_{0}$ is the first Lagrangian regular point at the right of 0 and $r_{i}<r_{i+1}$ for any $i \in \mathbb{Z}$. The next proposition claims that between two consecutive regular points, clusters may also be indexed by $\mathbb{Z}$.

Proposition 1. - Suppose that the initial potential $\psi_{0}$ is a stable Lévy process with index $\alpha \in(1 / 2,1)$ which is not completely asymmetric. Then, with probability one, there exists a unique sequence $\left(e_{i} ; i \in \mathbb{Z}\right)$ of (random) increasing maps

$$
e_{i}: \mathbb{Z} \rightarrow\left(r_{i}, r_{i+1}\right)
$$

such that

(i) the range of the sequence $\left(e_{i}(n) ; n \in \mathbb{Z}\right)$ is exactly the set of Eulerian shock points in $\left(r_{i}, r_{i+1}\right)$,

$$
\left\{\begin{array}{l}
u\left(e_{i}(n)\right)>0 \quad \text { for any } n<0 \\
u\left(e_{i}(n)\right)<0 \text { for any } n \geqslant 0
\end{array}\right.
$$

Moreover, $r_{i}=\lim _{n \downarrow-\infty} e_{i}(n)$ and $r_{i+1}=\lim _{n \uparrow \infty} e_{i}(n)$ a.s.

Recall that the velocity of a cluster located at $x$ is (according to the conservation of masses and momenta)

$$
v(x)=-\frac{\psi_{0}(a(x))-\psi_{0}(a(x-))}{a(x)-a(x-)}=\frac{u(a(x))+u(a(x-))}{2} .
$$


The following corollary ensures that the velocity of the clusters is positive (negative) at the right (left) of a regular point. Moreover, regular points are exactly the points where $u=0$.

COROLlary 1.1. - With probability one,

$$
\left\{\begin{array}{l}
v\left(e_{i}(n)\right)>0 \quad \text { for any } n<0, \\
v\left(e_{i}(n)\right)<0 \quad \text { for any } n>0,
\end{array}\right.
$$

and $\mathcal{R}=\{r ; u(r)=0\}$.

In the next theorem we claim that $\mathcal{R}$ is a regenerative set and that the turbulence has evolved (up to time 1) independently at the right and the left of a regular point. Physically, this property is quite intuitive. Regular points are particles with initial velocity zero, which have not been perturbed up to time 1 . So, there has been no interaction between the particles at the left of a regular point and those at its right. Mathematically, this property appears naturally as well. It is easily seen, mainly using splitting times (see [11] for a short introduction to splitting times) and the fact that $\psi_{0}$ has stationary increments, that $u$ is a (homogeneous) simple Markov process. If we knew that $u$ is a strong Markov process, the equality $\mathcal{R}=\{r \in \mathbb{R} ; u(r)=0\}$ would ensure the regenerative property of $\mathcal{R}$. Yet, we cannot conclude directly that $\mathcal{R}$ is regenerative, because we do not know whether $u$ is strongly Markovian or not.

By stationarity, we just state the regenerative property for $r_{0}$ that is the first regular point at the right of 0 .

THEOREM 2. - Suppose that the initial potential $\psi_{0}$ is a stable Lévy process with index $\alpha \in(1 / 2,1)$, that is not completely asymmetric. Then, the processes $\left(\psi_{0}\left(r_{0}+x\right)-\right.$ $\left.\psi_{0}\left(r_{0}\right) ; x \geqslant 0\right)$ and $\left(\psi_{0}\left(r_{0}-x\right) ; x \geqslant 0\right)$ are independent.

As a consequence, the processes $\left(u\left(r_{0}+x\right) ; x \geqslant 0\right)$ and $\left(u\left(r_{0}-x\right) ; x \geqslant 0\right)$ are independent and $\mathcal{R}$ is a regenerative set.

Let us now prove these results.

\subsection{Regular points form a discrete set}

The proof of Theorem 1 is closely connected to the proof of Theorem 4 in [6]. In particular, we may refer to [6] for some technical arguments.

Thanks to the stationarity of the initial potential, we just have to prove that

$$
\operatorname{card}\{\mathcal{R} \cap[0,1]\}<\infty \text { a.s. }
$$

Fix $\varepsilon>0$. If we prove that there exists a constant $K>0$ such that

$$
\mathbb{P}(\mathcal{R} \cap[0, \varepsilon] \neq \emptyset) \leqslant K \varepsilon,
$$

then by stationarity, $\mathbb{P}(\mathcal{R} \cap[a, a+\varepsilon] \neq \emptyset) \leqslant K \varepsilon$ for any $a \in \mathbb{R}$ and thanks to Fubini's Theorem

$$
\mathbb{E}(\operatorname{card}\{k=0, \ldots,[1 / \varepsilon] ; \mathcal{R} \cap[k \varepsilon,(k+1) \varepsilon] \neq \emptyset\}) \leqslant 2 K,
$$


which implies in particular that $\operatorname{card}\{\mathcal{R} \cap[0,1]\}<\infty$ a.s.

Bertoin ([6], Lemma 5) has proved that if $r \in \mathcal{R}$ then $\psi_{0}$ is continuous at $r$ and $r=a(r)$ (which means that $r$ had an initial velocity zero and has not been involved in the turbulence, so it stayed at the same location). In particular, we deduce from the definition of $a$ that a.s.

$$
\psi_{0}(r+h)-\frac{1}{2} h^{2} \leqslant \psi_{0}(r) \quad \forall h \in \mathbb{R}
$$

and hence that

$$
\{\mathcal{R} \cap[0, \varepsilon] \neq \emptyset\} \subset\left\{\exists r \in[0, \varepsilon] / \forall h \in \mathbb{R} ; \psi_{0}(r \pm h)-\psi_{0}(r) \leqslant \frac{1}{2} h^{2}\right\} .
$$

Evaluating the probability of the right-hand event involves the maximum of the inhomogeneous Markov process $\left\{\psi_{0}(r+h)-h^{2} / 2, h \in \mathbb{R}\right\}$, which is not convenient. We will thus replace this inhomogeneous Markov process by an homogeneous one in replacing the quadratic term by a $\beta$-stable subordinator $\sigma$ (remember it is a $\beta$-stable Lévy process with no negative jumps) fulfilling $\sigma(h) \geqslant h^{2} / 2$ with a probability at least $1-\delta$. We first state and prove a lemma that ensures the existence of such subordinators. We then see how to use them.

LEMMA 1. - For any $\frac{1}{2}<\beta<1$, consider a $\beta$-stable subordinator $\sigma$ with Laplace transform

$$
\mathbb{E}\left(\exp \left(-\lambda \sigma_{t}\right)\right)=\exp \left(-t c \lambda^{\beta}\right) .
$$

Then, for any fixed $\delta>0$, the parameter $c$ can be chosen such that the event

$$
\left\{\sigma(t+h)-\sigma(t) \geqslant h^{2}, \forall t \in[0,1], h \in(0,2]\right\}
$$

has a probability larger than $1-\delta$.

Proof. - This proof is adapted from Theorem 1 in [14]. Denote, for any integer $k, j, q$ such that $0 \leqslant j \leqslant 2^{q+1}$ and $1 \leqslant k \leqslant q$,

$$
A_{j k q}=\left\{\sigma\left((j+k) / 2^{q}\right)-\sigma\left(j / 2^{q}\right) \leqslant\left(\frac{k+2}{2^{q}}\right)^{2}\right\}
$$

and

$$
A=\bigcup_{q=1 . . \infty ; j=0 . .2^{q+1} ; k=1 . . q} A_{j k q} .
$$

We want to chose the parameter $c$ such that the event $A$ has a probability less than $\delta$. Thanks to the scaling property of $\sigma$ and the stationarity of its increments we have

$$
\mathbb{P}\left(A_{j k q}\right)=\mathbb{P}\left(\sigma(1) \leqslant\left(\frac{2^{q}}{k}\right)^{1 / \beta}\left(\frac{k+2}{2^{q}}\right)^{2}\right) \leqslant \mathbb{P}\left(\sigma(1) \leqslant 9 k^{2-1 / \beta} 2^{-q(2-1 / \beta)}\right) .
$$

Using the Markov inequality

$$
\mathbb{P}(\sigma(1) \leqslant x) \leqslant \mathrm{e}^{x .1 / x} \mathbb{E}\left(\mathrm{e}^{-\sigma(1) / x}\right) \leqslant \mathrm{e} \cdot \mathrm{e}^{-c x^{-\beta}},
$$


we get setting $\rho=2 \beta-1 \in(0,1)$

$$
\begin{aligned}
\mathbb{P}(A) & \leqslant \sum_{q=1}^{\infty} \sum_{j=0}^{2^{q+1}} \sum_{k=1}^{q} \mathbb{P}\left(A_{j k q}\right) \\
& \leqslant \sum_{q=1}^{\infty} \sum_{j=0}^{2^{q+1}} \sum_{k=1}^{q} \mathrm{e} \cdot \exp \left(-9^{-\beta} c 2^{q \rho} k^{-\rho}\right) \\
& \leqslant \mathrm{e} \sum_{q=1}^{\infty} q 2^{q+1} \exp \left(-9^{-\beta} c\left(2^{q} / q\right)^{\rho}\right) .
\end{aligned}
$$

So, if we choose $c$ large enough, we obtain $\mathbb{P}(A) \leqslant \delta$. We will check now that we have

$$
\sigma(t+h)-\sigma(t) \geqslant h^{2} \quad \forall t \in[0,1], h \in(0,2],
$$

on the event $A^{c}$. Given $0<h \leqslant 2, t \in[0,1]$, let $q, j, k$ be such that

$$
\frac{1}{2^{q-1}}<h \leqslant \frac{q}{2^{q}} \text { and } \quad \frac{j-1}{2^{q}}<t \leqslant \frac{j}{2^{q}}<\frac{j+k}{2^{q}} \leqslant t+h<\frac{j+k+1}{2^{q}} .
$$

We have, on $A^{c}$

$$
\sigma(t+h)-\sigma(t) \geqslant \sigma\left((j+k) / 2^{q}\right)-\sigma\left(j / 2^{q}\right)>\left(\frac{k+2}{2^{q}}\right)^{2}>h^{2},
$$

which concludes the proof of the lemma.

We are now ready to establish Theorem 1.

Proof of Theorem 1. - Fix $0<\delta<1$. Thanks to Lemma 1, we can choose two $\beta$-stable subordinators $\sigma^{+}$and $\sigma^{-}$with index $\frac{1}{2}<\beta<\alpha$, which satisfy the following conditions: $\sigma^{+}, \sigma^{-}$and $\psi_{0}$ are independent and the event

$$
A_{\delta}=\left\{\sigma^{+/-}(t+h)-\sigma^{+/-}(t) \geqslant h^{2}, \forall t \in[0,1], h \in[0,2]\right\}
$$

has probability larger than $1-\delta$.

Recall that we want to estimate the probability of the event

$$
B_{\varepsilon}=\left\{\exists r \in[0, \varepsilon] / \forall h \in \mathbb{R} ; \psi_{0}(r \pm h)-\psi_{0}(r) \leqslant h^{2} / 2\right\} .
$$

We deduce from the independence of $\sigma^{+}, \sigma^{-}$and $\psi_{0}$ that

$$
\mathbb{P}\left(B_{\varepsilon}\right)=\mathbb{P}\left(B_{\varepsilon} \cap A_{\delta}\right) / \mathbb{P}\left(A_{\delta}\right) .
$$

In particular, we just need to prove that

$$
\mathbb{P}\left(B_{\varepsilon} \cap A_{\delta}\right)=\mathrm{O}(\varepsilon) .
$$


Let us consider $\omega \in B_{\varepsilon} \cap A_{\delta}$ and a regular point $r=r(\omega) \in[0, \varepsilon]$. Recall that the initial potential $\psi_{0}$ then fulfills

$$
\psi_{0}(r \pm h)-\psi_{0}(r) \leqslant \frac{1}{2} h^{2} \quad \forall h \in \mathbb{R},
$$

and as a consequence

$$
\psi_{0}(x)-\frac{1}{2}(r-x)^{2} \leqslant \psi_{0}(r) \text { for } \varepsilon-1 \leqslant x \leqslant r .
$$

So, applying the condition on the growth of $\sigma^{-}$with $t=1-\varepsilon+r$ and $h=r-x$, we get

$$
\psi_{0}(x)+\sigma^{-}(1-\varepsilon+x)-\sigma^{-}(1-\varepsilon+r) \leqslant \psi_{0}(r) \text { for } \varepsilon-1 \leqslant x \leqslant r .
$$

In particular, the Lévy process

$$
Y^{-}(x)=\psi_{0}(x)+\sigma^{-}(1-\varepsilon+x) \text { for } x \geqslant \varepsilon-1
$$

reaches a new maximum at $r \in[0, \varepsilon]$. Let $\tau$ denote the first time after 0 where $Y^{-}$reaches a new maximum and write $\eta=r-\tau \in[0, \varepsilon]$. The inequality

$$
\psi_{0}(r+h)-\psi_{0}(r) \leqslant \frac{1}{2} h^{2} \quad \forall h>0
$$

implies

$$
\psi_{0}(\tau+\eta+h)-\psi_{0}(\tau+\eta) \leqslant \frac{1}{2} h^{2} \leqslant \sigma^{+}(\eta+h)-\sigma^{+}(\eta) \quad \forall h \in[0,2-\eta] .
$$

So the process

$$
Y^{+}(x)=\psi_{0}(\tau+x)-\psi_{0}(\tau)-\sigma^{+}(x) \text { for } x \geqslant 0
$$

does not reach a new maximum in $(\varepsilon, 1+\varepsilon)$. Moreover, it is a Lévy process independent of $Y^{-}$since $\tau$ is a stopping time. Putting the pieces together, one obtains that

$$
\mathbb{P}\left(B_{\varepsilon} \cap A_{\delta}\right) \leqslant \mathbb{P}_{1}(\varepsilon) \times \mathbb{P}_{2}(\varepsilon),
$$

where

$$
\left\{\begin{array}{l}
\mathbb{P}_{1}=\mathbb{P}\left(Y^{-} \text {reaches a new max in }[0, \varepsilon]\right), \\
\mathbb{P}_{2}=\mathbb{P}\left(Y^{+} \text {does not reach a new max in }[\varepsilon, 1+\varepsilon]\right) .
\end{array}\right.
$$

Now, the proof follows the same line as Lemma 7 in [6]. We first give an upper bound to $\mathbb{P}_{2}(\varepsilon)$. Like in [6] we have by the fluctuation theory for Lévy processes

$$
\mathbb{P}_{2}(\varepsilon)=\mathrm{O}(\phi(1 / \varepsilon))^{-1}
$$


where

$$
\phi(1 / \varepsilon)=\exp \left(\int_{0}^{\infty} \frac{\mathrm{e}^{-s}-\mathrm{e}^{-s / \varepsilon}}{s} \mathbb{P}\left(Y^{+}(s) \geqslant 0\right) \mathrm{d} s\right) .
$$

We evaluate the latter quantity using the scaling property

$$
\begin{aligned}
\mathbb{P}\left(Y^{+}(s) \geqslant 0\right) & =\mathbb{P}\left(\psi_{0}(s) \geqslant \sigma^{+}(s)\right) \\
& =\mathbb{P}\left(\psi_{0}(1) \geqslant s^{\gamma} \sigma^{+}(1)\right) \\
& =\mathbb{P}\left(\psi_{0}(1) \geqslant 0\right)-\mathbb{P}\left(0 \leqslant \psi_{0}(1)<s^{\gamma} \sigma^{+}(1)\right),
\end{aligned}
$$

where $\gamma=1 / \beta-1 / \alpha>0$. We get from the independence of $\psi_{0}$ and $\sigma^{+}$

$$
\mathbb{P}\left(0 \leqslant \psi_{0}(1)<s^{\gamma} \sigma^{+}(1)\right)=\int_{y \geqslant 0} \mathbb{P}\left(\psi_{0}(1) \in \mathrm{d} y\right) \mathbb{P}\left(\sigma^{+}(1)>y / s^{\gamma}\right) .
$$

For $s<1$, Markov inequality yields

$$
\mathbb{P}\left(\sigma^{+}(1)>y / s^{\gamma}\right) \leqslant\left(y^{-1 / 2} s^{\gamma / 2} \mathbb{E}\left(\sqrt{\sigma^{+}(1)}\right)\right) \wedge 1,
$$

so writing $k=\mathbb{E}\left(\sqrt{\sigma^{+}(1)}\right)<\infty$ (since $\sigma^{+}$is $\beta$-stable with $\beta>1 / 2$ )

$$
\begin{aligned}
\mathbb{P}\left(0 \leqslant \psi_{0}(1)<s^{\gamma} \sigma^{+}(1)\right) & \leqslant \int_{y \geqslant 0} \mathbb{P}\left(\psi_{0}(1) \in \mathrm{d} y\right)\left(\frac{k s^{\gamma / 2}}{\sqrt{y}} \wedge 1\right) \\
& \leqslant \int_{0}^{k^{2} s^{\gamma}} \mathbb{P}\left(\psi_{0}(1) \in \mathrm{d} y\right)+\int_{y \geqslant k^{2} s^{\gamma}} \frac{\mathbb{P}\left(\psi_{0}(1) \in \mathrm{d} y\right)}{\sqrt{y}} k s^{\gamma / 2} \\
& \leqslant K k^{2} s^{\gamma}+K k s^{\gamma / 2} \int_{k^{2} s^{\gamma}}^{1} \frac{\mathrm{d} y}{\sqrt{y}}+k s^{\gamma / 2} \int_{1}^{\infty} \mathbb{P}\left(\psi_{0}(1) \in \mathrm{d} y\right) \\
& \leqslant \text { constant. } s^{\gamma / 2},
\end{aligned}
$$

where $K=\sup _{y \in[0,1]}\left(\mathbb{P}\left(\psi_{0}(1) \in \mathrm{d} y\right) / \mathrm{d} y\right)$ is a finite constant, since stable laws have continuous densities. With the notation $\rho=\mathbb{P}\left(\psi_{0}(1) \geqslant 0\right)$ we deduce that

$$
\phi(1 / \varepsilon) \geqslant \varepsilon^{-\rho} \underbrace{\exp \left(-\int_{0}^{\infty} \frac{\mathrm{e}^{-s}}{s} \mathbb{P}\left(0 \leqslant \psi_{0}(1)<s^{\gamma} \sigma^{+}(1)\right) \mathrm{d} s\right)}_{\text {constant }>0}
$$

and we conclude that

$$
\mathbb{P}_{2}(\varepsilon)=\mathrm{O}\left(\varepsilon^{\rho}\right) .
$$

Let us now evaluate the probability that $Y^{-}$reaches a new maximum in $[0, \varepsilon]$. As in [6] we introduce the time-reversed process

$$
Z(x)=Y^{-}(\varepsilon-x-)-Y^{-}(\varepsilon) \text { for } x \in[0,1] .
$$


This is a Lévy process, which has the same law as $-\psi_{0}(x)-\sigma^{-}(x)$. We thus have $\mathbb{P}\left(Y^{-}\right.$reaches a new max in $\left.[0, \varepsilon]\right)=\mathbb{P}(Z$ does not reach a new max in $(\varepsilon, 1+\varepsilon])$ and

$$
\begin{aligned}
\mathbb{P}(Z(s) \geqslant 0) & =\mathbb{P}\left(\psi_{0}(1) \leqslant 0\right)-\mathbb{P}\left(-s^{\gamma} \sigma^{-}(1)<\psi_{0}(1) \leqslant 0\right) \\
& =1-\rho-\mathbb{P}\left(-s^{\gamma} \sigma^{-}(1)<\psi_{0}(1) \leqslant 0\right) .
\end{aligned}
$$

We deduce from what has previously been done for $Y^{+}$, the inequality

$$
\mathbb{P}_{1}(\varepsilon)=\mathrm{O}\left(\varepsilon^{1-\rho}\right)
$$

which allows us to conclude that (3) holds and this ends the proof of Theorem 1.

\subsection{Precisions on the turbulence}

This subsection is broken into three lemmas. Putting the pieces together, one deduces the results stated in Proposition 1. The first lemma deals with the structure of Eulerian shock points.

LEMMA 2. - With probability one, Eulerian shock points between two consecutive regular points form a (random) increasing sequence indexed by $\mathbb{Z}$.

Proof. - The lemma exactly means that a.s. Lagrangian regular points are the only accumulation points of the range of the inverse of the Lagrangian function $a$. So, let us consider a right accumulation point $d$ of the range of $a$ (similar arguments apply to left accumulation points). We will prove that $d$ is a regular point. The line of the proof is the following: we first check that $\psi_{0}$ is a.s. continuous at $d$, we then prove that a.s. $a(d)=d$ and $a$ is continuous at $d$, and finally we conclude.

(i) Potential $\psi_{0}$ is continuous at $d$. Suppose that $d$ is a jump time for $\psi_{0}$. We will check that we then have $a(d)=d$, and that this cannot agree with our assumption. Let $d_{n}=a\left(x_{n}\right)$ be a sequence decreasing to $d$ and set $c=\lim _{n \rightarrow \infty} x_{n}$. The right continuity of $a$ implies that $a(c)=d$. The identity $d_{n}=a\left(x_{n}\right)$ entails that

$$
\left(\psi_{0}\left(d_{n}-\right) \vee \psi_{0}\left(d_{n}\right)\right)-\frac{1}{2}\left(x_{n}-d_{n}\right)^{2} \geqslant \psi_{0}(d)-\frac{1}{2}\left(x_{n}-d\right)^{2},
$$

so

$$
\frac{\left(\psi_{0}\left(d_{n}-\right) \vee \psi_{0}\left(d_{n}\right)\right)-\psi_{0}(d)}{d_{n}-d} \geqslant \frac{d_{n}+d-2 x_{n}}{2}
$$

and finally

$$
\limsup _{h \downarrow 0} \frac{\psi_{0}(d+h)-\psi_{0}(d)}{h} \geqslant d-c .
$$

Consider now $d<d_{n}$ and $c<x_{n}$. The identity $d_{n}=a\left(x_{n}\right)$ yields

$$
\begin{aligned}
\psi_{0}\left(d_{n}+h\right)-\left(\psi_{0}\left(d_{n}-\right) \vee \psi_{0}\left(d_{n}\right)\right) & \leqslant \frac{1}{2}\left(d_{n}+h-x_{n}\right)^{2}-\frac{1}{2}\left(d_{n}-x_{n}\right)^{2} \\
& \leqslant h\left(d_{n}-x_{n}+h / 2\right) .
\end{aligned}
$$


Using the right continuity of $\psi_{0}$, we get

$$
\psi_{0}(d+h)-\psi_{0}(d) \leqslant h(d-c)+\frac{1}{2} h^{2}
$$

which implies that

$$
\limsup _{h \downarrow 0} \frac{\psi_{0}(d+h)-\psi_{0}(d)}{h} \leqslant d-c .
$$

We deduce from (5) and (6) that

$$
\limsup _{h \downarrow 0} \frac{\psi_{0}(d+h)-\psi_{0}(d)}{h}=d-c .
$$

A result of Khintchine (see Theorem VIII.5 in [3] for an accessible reference) give the following information on the local behavior of $\psi_{0}$ :

$$
\text { for any } \beta>0, \quad \text { a.s. } \limsup _{h \downarrow 0} \frac{\psi_{0}(h)}{h^{\beta}}= \begin{cases}0 & \text { if } \alpha<1 / \beta, \\ \infty & \text { if } \alpha \geqslant 1 / \beta .\end{cases}
$$

Since the set of discontinuities of $\psi_{0}$ is a countable set of stopping times; one deduces from (7) that a.s.

$$
\limsup _{h \downarrow 0} \frac{\psi_{0}(d+h)-\psi_{0}(d)}{h}=d-c=0,
$$

i.e. $a(d)=d$.

Now, (7) ensures that a.s. for any time $d$ of discontinuity of $\psi_{0}$

$$
\limsup _{h \downarrow 0} \frac{\psi_{0}(d+h)-\psi_{0}(d)}{h^{2}}=\infty,
$$

which makes the equality $d=a(d)$ impossible. We have proved that $\psi_{0}$ is a.s. continuous at any right-accumulation point $d$.

(ii) A.s., for any right accumulation point $d$, we have $a(d)=d$. The argument of Lemma 5 in [6] shows that with probability 1 at any point $c$ of the range of $a$ where $\psi_{0}$ is continuous, we have

$$
\left\{\begin{array}{l}
c=a(c) \\
x \neq c \Longrightarrow a(x) \neq a(c) .
\end{array}\right.
$$

In particular $a(d)=d$ and $a$ is strictly increasing in $d$.

(iii) The inverse of the Lagrangian function a is continuous at $d$. We first check that if $a(d-) \neq a(d)=d$, then $a(d-)$ is a time of negative jump for $\psi_{0}$. It is known that $\psi_{0}=S^{(1)}-S^{(2)}$, where $S^{(1)}$ and $S^{(2)}$ are two independent $\alpha$-stable subordinators (recall that a subordinator is a Lévy process with no negative jump). Suppose that $t=a(d-)$ is not the time of a negative jump for $\psi_{0}$. Then

$$
S^{(1)}(t+h)-S^{(1)}(t) \leqslant S^{(2)}(t+h)-S^{(2)}(t)-h(\underbrace{t-d}_{<0}-h / 2) .
$$


A result of Fristedt [10] claims that for any $\alpha$-stable subordinator $S$ we have a.s.

$$
\text { for any } t \in \mathbb{R}, \quad \liminf _{h \rightarrow 0} \frac{S(t+h)-S(t)}{h^{1 / \alpha}}<\infty,
$$

from which follows that a.s.

$$
\liminf _{h \rightarrow 0} \frac{S^{(2)}(t+h)-S^{(2)}(t)}{h}=0 .
$$

As a consequence, there exists some small $h$ such that $S^{(1)}(t+h)-S^{(1)}(t)<0$, which is absurd. So, $t=a(d-)$ is a time of negative jump for $\psi_{0}$.

Since $a(d)=d$ we have the equality

$$
\psi_{0}(t-)=\psi_{0}(d)+\frac{1}{2}(d-t)^{2}
$$

which leads us to consider the set $\mathcal{T}$ of time $\tau$ such that there exists a negative jump of $\psi_{0}$, say $T<\tau$, checking

$$
\psi_{0}(T-)=\psi_{0}(\tau)+\frac{1}{2}(T-\tau)^{2} .
$$

Since negative jumps of $\psi_{0}$ form a countable set of stopping times, one deduces that the set $\mathcal{T}$ is a countable set of stopping times $\tau$. In particular (7) ensures that a.s. for any $\tau \in \mathcal{T}$

$$
\limsup _{h \downarrow 0} \frac{\psi_{0}(\tau+h)-\psi_{0}(\tau)}{h^{2}}=\infty,
$$

which impedes $\tau$ to be a right accumulation point of the range of $a$. In particular, $d \in \mathcal{T}$ is impossible a.s. and $a(d-)=a(d)=d$.

(iv) Conclusion. Recall that (8) ensures that $a$ is strictly increasing at $d$, and since $a$ is continuous at $d$, one deduces that $d$ is a left and right accumulation point in the range of $a$, i.e. $d$ is regular.

We now turn our attention to the local behavior of $\psi_{0}$ at a point of the range of $a$.

LEMMA 3. - With probability one we have:

(i) Regular points are exactly the points of the range of $x \rightarrow a(x)$ where $\psi_{0}$ is continuous.

(ii) Suppose that $x$ is not a regular point. If $a(x)>x$, then $a(x)$ is a time of positive jump for $\psi_{0}$, whereas it is a time of negative jump, if $a(x)<x$.

Proof. -

(i) We have seen in the proof of the previous Lemma that $\psi_{0}$ is continuous at any right accumulation point of the range of $a$. In particular, $\psi_{0}$ is continuous at any regular point. Conversely, if $\psi_{0}$ is continuous at a point $c$ of the range of $a$, then (8) ensures that a.s. $c=a(c)$ and that $a$ is strictly increasing at $c$. Since $a$ is right continuous, one deduces that $c$ is a.s. a right accumulation point of the range of $a$, which means using the previous lemma that a.s. $c$ is Lagrangian regular. 
(ii) The first part of the lemma ensures that a point $a(x)$ that is not a regular point, is a time of jump for $\psi_{0}$. Since the times of jump of $\psi_{0}$ form a countable set of stopping times, one see from (7) that with probability one for any time $T$ of jump of $\psi_{0}$

$$
\underset{h \downarrow 0}{\limsup } \frac{\psi_{0}(T+h)-\psi_{0}(T)}{h}=0 \quad \text { and } \quad \limsup _{h \downarrow 0} \frac{\psi_{0}(T-h)-\psi_{0}(T-)}{h}=0 .
$$

If $a(x)$ is a time of positive jump of $\psi_{0}$ then

$$
\psi_{0}(a(x)+h)-\psi_{0}(a(x)) \leqslant \frac{1}{2}(a(x)+h-x)^{2}-\frac{1}{2}(a(x)-x)^{2},
$$

i.e.

$$
\frac{\psi_{0}(T+h)-\psi_{0}(T)}{h} \leqslant a(x)-x+h / 2
$$

and

$$
\limsup _{h \downarrow 0} \frac{\psi_{0}(T+h)-\psi_{0}(T)}{h}=0 \leqslant a(x)-x .
$$

We deduce in the same way that if $a(x)$ is a time of a negative jump of $\psi_{0}$ then $a(x) \leqslant x$. The proof of the lemma is complete.

In the final lemma, we focus on the behavior of $u$.

LEMMA 4. - With probability one, the set $\mathcal{R}$ of regular points is exactly the set of points where $u$ has velocity 0 . Moreover, when $x$ increases from a given regular point to the next, the velocity $u(x)$ is a.s. first positive and then negative.

As a consequence, when $x$ increases the velocity of the clusters between two consecutive regular points is a.s. first positive and then negative.

Proof. - Bertoin (Lemma 5 in [6]) has proved that with probability one, any regular point has velocity 0 . Conversely, if $a(r)=r, r$ cannot be a time of jump for $\psi_{0}$ since (7) ensures that a.s. for any time $T$ of jump of $\psi_{0}$

$$
\underset{h \downarrow 0}{\limsup } \frac{\psi_{0}(T+h)-\psi_{0}(T)}{h^{2}}=\infty .
$$

We deduce from the previous lemma that $r$ is regular.

Between two regular points, $u$ is a tooth path, made of pieces of line of slope 1 , split by negative jumps (shocks). In particular, $u$ is continuous at any point of increase, and $u$ cannot go from negative value to positive value without crossing a regular point. We shall prove now that $u$ cannot stay always positive or negative between two regular points. Let us prove for example that $u$ cannot stay always positive between $r_{-1}$ and $r_{0}$ (recall that $r_{0}$ is the first regular point at the right of 0 , whereas $r_{-1}$ is the first one at the left of 0 ). Define

$$
T=\inf \left\{t>0 ; \psi_{0}(z)-\psi_{0}(t) \leqslant \frac{1}{2}(z-t)^{2}, \forall z \leqslant t\right\}
$$


Obviously, $T$ is a stopping time, such that $T \leqslant r_{0}$. We first prove that if $u>0$ on $\left(r_{-1}, r_{0}\right)$, then $T=r_{0}$. Suppose that $T<r_{0}$. There exists $y \in\left(r_{-1}, r_{0}\right)$ such that $T \in(a(y-), a(y)]$. Call $\mathcal{C}_{y}$ the parabola defined by $\left\{\frac{1}{2}(z-y)^{2}+C ; z \in \mathbb{R}\right\}$, where $C$ is chosen such that $\mathcal{C}_{y}$ is above the graph of $\psi_{0}$ but touches it at $a(y-)$ and $a(y)$. Then, geometrically, $\mathcal{C}_{y}$ is strictly above the half-parabola $\left\{\psi_{0}(T)+\frac{1}{2}(z-T)^{2} ; z<T\right\}$, because $T \leqslant a(y)<y($ remember that $u(y)>0)$ and $\mathcal{C}_{y}$ is above $\psi_{0}(T)$. In particular, $a(y-)$ is strictly above this half-parabola, what contradicts the definition of $T$. So $T=r_{0}$. Yet, $T$ is a stopping time, so $\psi_{0}(T+)-.\psi_{0}(T)$ has the same law as $\psi$. We deduce that a.s. $T$ is not a regular point, so $u$ cannot be positive between $r_{-1}$ and $r_{0}$. We finally deduce that after a regular point, the velocity $u$ is first positive and then negative, with one and only one change of sign.

Consider an Eulerian shock point $x$. The identity $v(x)=(u(a(x))+u(a(x-))) / 2$ ensures that the velocity of the clusters between two consecutive regular points is a.s. first positive and then negative (when $x$ increases).

Remark. - Bertoin (Theorem 3 in [6]) has proved that a local maximum of $\psi_{0}$ has a positive probability to be a regular point. Conversely, one may easily see from the previous lemma, that a regular point is a.s. a local maximum of $\psi_{0}$.

\subsection{Regenerative property of regular points}

The idea of the proof of Theorem 2 is the following. We consider an approximation $T_{\delta, \varepsilon}$ of the first regular point $r_{0}$ at the right of 0 that satisfies a Markov type property and we then obtain the regeneration property for $r_{0}$ by taking the limits.

Let us first introduce some notations. Let $\mathcal{T}=\left\{t \in \mathbb{R}^{+}: \psi_{0}(t) \neq \psi_{0}(t-)\right\}$ denote the set of positive jump points of the initial potential $\psi_{0}$, and write

$$
\begin{aligned}
\psi_{0}^{a} & =\left\{\psi_{0}(a+t)-\psi_{0}(a), t \in \mathbb{R}\right\}, \\
\psi_{0-}^{a} & =\left\{\psi_{0}(t), t \leqslant a\right\}, \quad \psi_{0+}^{a}=\left\{\psi_{0}(a+t)-\psi_{0}(a), t \geqslant 0\right\},
\end{aligned}
$$

and

$$
\mathcal{A}_{(a, b)}^{\varepsilon}=\left\{g \in \mathbb{D}: g(s) \leqslant f^{\varepsilon}(s) \forall s \in(a, b)\right\},
$$

where

$$
f^{\varepsilon}(s)=\mathbf{1}_{s<0}\left(\frac{1}{2}(s-\varepsilon)^{2}-\frac{1}{2} \varepsilon^{2}\right)+\mathbf{1}_{s \geqslant 0}\left(\frac{1}{2} s^{2}\right)
$$

and $\mathbb{D}$ is the space of right continuous with left limits functions $g: \mathbb{R} \rightarrow \mathbb{R}$. We will consider as an approximation of the first positive regular point, the time

$$
T_{\delta, \varepsilon}=\inf \left\{\tau \in \mathcal{T}: \psi_{0}(\tau)-\psi_{0}(\tau-)<-\delta \text { and } \psi_{0}^{\tau} \in \mathcal{A}_{\mathbb{R}}^{\varepsilon}\right\}
$$

The following lemma ensures that $T_{\delta, \varepsilon}$ tends to $r_{0}$ when we first let $\delta \downarrow 0$ and then $\varepsilon \downarrow 0$.

LEMMA 5. - With probability one

(i) When $\delta$ decreases to $0, T_{\delta, \varepsilon}$ decreases to a time $T_{\varepsilon}$ fulfilling $T_{\varepsilon} \leqslant r_{0}$.

(ii) When $\varepsilon$ decreases to $0, T_{\varepsilon}$ increases to $r_{0}$. 


\section{Proof. -}

(i) In the notation of proposition 1, consider the sequence of Eulerian shock points $\left(e_{0}(n) ; n<0\right)$ that decreases to $r_{0}$ when $n$ tends to $-\infty$. Remember that a.s. $r_{0}=a\left(r_{0}\right)$, and $u\left(e_{0}(n)\right)>0$ for $n<0$. So, there exists $N \in \mathbb{N}$ such that $0<e_{0}(n)-a\left(e_{0}(n)\right) \leqslant \varepsilon$ for $n \leqslant-N$. In particular, $\psi_{0}^{e_{0}(-N)} \in \mathcal{A}_{\mathbb{R}}^{\varepsilon}$. Moreover, Lemma 4 ensures that $e_{0}(-N)$ is a time of negative jump and if we choose $\delta$ small enough $\psi_{0}\left(e_{0}(-N)\right)-\psi_{0}\left(e_{0}(-N-)\right)<-\delta$. This implies that

$$
T_{\varepsilon} \leqslant T_{\delta, \varepsilon} \leqslant e_{0}(-N)
$$

Finally, we let $\delta \downarrow 0$, and we deduce that a.s. $T_{\varepsilon} \leqslant r_{0}$.

(ii) The function $f^{\varepsilon}$ is increasing with $\varepsilon$, which makes the condition $\mathcal{A}_{\mathbb{R}}^{\varepsilon}$ more and more restrictive when $\varepsilon$ decreases to 0 . So $T^{\varepsilon}$ increases to, say, $T \leqslant r_{0}$ when $\varepsilon$ decreases to 0 . The process $\psi_{0}^{T}$ fulfills the condition $\mathcal{A}_{\mathbb{R}}^{0}$. In particular, we see from (7) that $T$ is not a time of jump for $\psi$. So Lemma 3(i) ensures that $T$ must be regular and finally $T=r_{0}$.

The following lemma ensures that $T_{\delta, \varepsilon}$ is finite a.s.

LEMMA 6. - If one chooses $\delta$ small enough, then $\mathbb{P}\left(T_{\delta, \varepsilon}<\infty\right)=1$.

Proof. - The lemma relies mainly on the $0-1$ law of Kolmogorov. The previous lemma ensures that $T_{\varepsilon} \leqslant r_{0}$ a.s., so if one chooses $\delta$ small enough then $\mathbb{P}\left(T_{\delta, \varepsilon}<\infty\right)>0$. Call a $\delta, \varepsilon$-regular point (resp. local- $\delta, \varepsilon$-regular point), a point $\tau$ fulfilling the conditions

$$
\tau \in \mathcal{T}, \quad \psi_{0}(\tau)-\psi_{0}(\tau-)<-\delta \quad \text { and } \quad \psi_{0}^{\tau} \in \mathcal{A}_{\mathbb{R}}^{\varepsilon} \quad\left(\text { resp. } \psi_{0}^{\tau} \in \mathcal{A}_{(-1,1)}^{\varepsilon}\right) .
$$

The first $\delta, \varepsilon$-regular point at the right of 0 is $T_{\delta, \varepsilon}$ and let us denote for any $M>0, T_{l o c}^{M}$ the first local- $\delta, \varepsilon$-regular point at the right of $\mathrm{M}$.

Let $X_{i}$ denotes the pieces of path $\left(\psi_{0}(i+x)-\psi_{0}(i) ; x \in(0,1)\right)$. The sequence $\left(X_{i} ; i \in \mathbb{N}\right)$ is a sequence of i.i.d. variables. The existence of arbitrarily large local- $\delta$, $\varepsilon$-regular points is an event of the tail $\sigma$-field

$$
\bigcap_{n \in \mathbb{N}} \sigma\left(X_{i} ; i>n\right)
$$

In particular, the 0-1 law of Kolmogorov ensures that the existence of arbitrarily large local- $\delta, \varepsilon$-regular points has probability 0 or 1 . The identity in law $\psi_{0}(M+)-.\psi_{0}(M) \sim$ $\psi_{0}$ leads us to

$$
\mathbb{P}\left(T_{\text {loc }}^{M}<\infty\right)=\mathbb{P}\left(T_{\text {loc }}^{0}<\infty\right) \geqslant \mathbb{P}\left(T_{\delta, \varepsilon}<\infty\right)>0,
$$

which implies in particular that there exists arbitrarily large local- $\delta, \varepsilon$-regular points with probability $>0$. This probability is then 1 . Moreover, a point $T_{l o c}^{M}$ has a positive probability (independent of $M$ ) to be a $\delta, \varepsilon$-regular point, so one deduces that there exists $\delta, \varepsilon$-regular point with probability one.

We now turn our attention to the Markov property type of $T_{\delta, \varepsilon}$. 
LEMMA 7. - In the above notation, $\psi_{0+}^{T_{\delta, \varepsilon}}$ and $\psi_{0-}^{T_{\delta, \varepsilon}}$ are independent.

Proof. - We want to prove that for any $(f, g) \operatorname{Borel}(\mathbb{D})$-measurable bounded functions the following equality holds

$$
\mathbb{E}\left(f\left(\psi_{0-}^{T_{\delta, \varepsilon}}\right) g\left(\psi_{0+}^{T_{\delta, \varepsilon}}\right)\right)=\mathbb{E}\left(f\left(\psi_{0-}^{T_{\delta, \varepsilon}}\right)\right) \mathbb{E}\left(g\left(\psi_{0+}^{T_{\delta, \varepsilon}}\right)\right) .
$$

Let $\left\{\tau_{1}, \ldots, \tau_{i}, \ldots\right\}$ denote the increasing sequence of positive times where the initial potential makes a jump of size less than $-\delta$. We have

$$
\begin{aligned}
\mathbb{E}\left(f\left(\psi_{0-}^{T_{\delta, \varepsilon}}\right) g\left(\psi_{0+}^{T_{\delta, \varepsilon}}\right)\right) & =\sum_{i=1}^{\infty} \mathbb{E}\left(f\left(\psi_{0-}^{\tau_{i}}\right) g\left(\psi_{0+}^{\tau_{i}}\right) ; T_{\delta, \varepsilon}=\tau_{i}\right) \\
& =\sum_{i=1}^{\infty} \mathbb{E}\left(f\left(\psi_{0-}^{\tau_{i}}\right) g\left(\psi_{0+}^{\tau_{i}}\right) ; \psi_{0}^{\tau_{i}} \in \mathcal{A}_{\mathbb{R}}^{\varepsilon} ; \psi_{0}^{\tau_{1}}, \ldots, \psi_{0}^{\tau_{i-1}} \notin \mathcal{A}_{\mathbb{R}}^{\varepsilon}\right) .
\end{aligned}
$$

We deduce from the convexity of $f^{\varepsilon}$ the identity

$$
\left\{\psi_{0}^{\tau_{i}} \in \mathcal{A}_{\mathbb{R}}^{\varepsilon} ; \psi_{0}^{\tau_{1}}, \ldots, \psi_{0}^{\tau_{i-1}} \notin \mathcal{A}_{\mathbb{R}}^{\varepsilon}\right\}=\left\{\psi_{0}^{\tau_{i}} \in \mathcal{A}_{\mathbb{R}}^{\varepsilon} ; \psi_{0}^{\tau_{1}}, \ldots, \psi_{0}^{\tau_{i-1}} \notin \mathcal{A}_{\left(-\infty, \tau_{i}\right)}^{\varepsilon}\right\} .
$$

Moreover, we know that each $\tau_{i}$ is a stopping time, so the processes $\psi_{0-}^{\tau_{i}}$ and $\psi_{0+}^{\tau_{i}}$ are independent, which ensures the next identities:

$$
\begin{aligned}
\mathbb{E}( & \left.f\left(\psi_{0-}^{T_{\delta, \varepsilon}}\right) g\left(\psi_{0+}^{T_{\delta, \varepsilon}}\right)\right) \\
= & \sum_{i=1}^{\infty} \mathbb{E}\left(f\left(\psi_{0-}^{\tau_{i}}\right) g\left(\psi_{0+}^{\tau_{i}}\right) ; \psi_{0}^{\tau_{i}} \in \mathcal{A}_{\left(-\infty, \tau_{i}\right)}^{\varepsilon} ; \psi_{0}^{\tau_{1}}, \ldots, \psi_{0}^{\tau_{i-1}} \notin \mathcal{A}_{\left(-\infty, \tau_{i}\right)}^{\varepsilon} ; \psi_{0}^{\tau_{i}} \in \mathcal{A}_{\left(\tau_{i}, \infty\right)}^{\varepsilon}\right) \\
= & \sum_{i=1}^{\infty} \mathbb{E}\left(f\left(\psi_{0-}^{\tau_{i}}\right) ; \psi_{0}^{\tau_{i}} \in \mathcal{A}_{\left(-\infty, \tau_{i}\right)}^{\varepsilon} ; \psi_{0}^{\tau_{1}}, \ldots, \psi_{0}^{\tau_{i-1}} \notin \mathcal{A}_{\left(-\infty, \tau_{i}\right)}^{\varepsilon}\right) \\
& \times \mathbb{E}\left(g\left(\psi_{0+}^{\tau_{i}}\right) ; \psi_{0}^{\tau_{i}} \in \mathcal{A}_{\left(\tau_{i}, \infty\right)}^{\varepsilon}\right) .
\end{aligned}
$$

The stationarity of $\psi_{0}$ yields

$$
\mathbb{E}\left(g\left(\psi_{0+}^{\tau_{i}}\right) ; \psi_{0}^{\tau_{i}} \in \mathcal{A}_{\left(\tau_{i}, \infty\right)}^{\varepsilon}\right)=\mathbb{E}\left(g\left(\psi_{0+}^{\tau_{j}}\right) ; \psi_{0}^{\tau_{j}} \in \mathcal{A}_{\left(\tau_{j}, \infty\right)}^{\varepsilon}\right)
$$

and

$$
\mathbb{P}\left(\psi_{0}^{\tau_{i}} \in \mathcal{A}_{\left(\tau_{i}, \infty\right)}^{\varepsilon}\right)=\mathbb{P}\left(\psi_{0}^{\tau_{j}} \in \mathcal{A}_{\left(\tau_{j}, \infty\right)}^{\varepsilon}\right) \quad \text { for any integer } i, j .
$$

Remember that

$$
\begin{aligned}
1 & =\mathbb{P}\left(T_{\delta, \varepsilon}<\infty\right) \\
& =\sum_{j=1}^{\infty} \mathbb{P}\left(\psi_{0}^{\tau_{j}} \in \mathcal{A}_{\left(-\infty, \tau_{j}\right)}^{\varepsilon} ; \psi_{0}^{\tau_{1}}, \ldots, \psi_{0}^{\tau_{j-1}} \notin \mathcal{A}_{\left(-\infty, \tau_{j}\right)}^{\varepsilon}\right) \mathbb{P}\left(\psi_{0}^{\tau_{j}} \in \mathcal{A}_{\left(\tau_{j}, \infty\right)}^{\varepsilon}\right),
\end{aligned}
$$

so we get with the preceding the following identities 


$$
\begin{aligned}
\mathbb{E}( & \left.f\left(\psi_{0-}^{T_{\delta, \varepsilon}}\right) g\left(\psi_{0+}^{T_{\delta, \varepsilon}}\right)\right) \\
= & \sum_{i=1}^{\infty} \mathbb{E}\left(f\left(\psi_{0-}^{\tau_{i}}\right) ; \psi_{0}^{\tau_{i}} \in \mathcal{A}_{\left(-\infty, \tau_{i}\right)}^{\varepsilon} ; \psi_{0}^{\tau_{1}}, \ldots, \psi_{0}^{\tau_{i-1}} \notin \mathcal{A}_{\left(-\infty, \tau_{i}\right)}^{\varepsilon}\right) \mathbb{P}\left(\psi_{0}^{\tau_{i}} \in \mathcal{A}_{\left(\tau_{i}, \infty\right)}^{\varepsilon}\right) \\
& \times \sum_{j=1}^{\infty} \mathbb{E}\left(g\left(\psi_{0+}^{\tau_{j}}\right) ; \psi_{0}^{\tau_{j}} \in \mathcal{A}_{\left(\tau_{j}, \infty\right)}^{\varepsilon}\right) \mathbb{P}\left(\psi_{0}^{\tau_{j}} \in \mathcal{A}_{\left(-\infty, \tau_{j}\right)}^{\varepsilon} ; \psi_{0}^{\tau_{1}}, \ldots, \psi_{0}^{\tau_{j-1}} \notin \mathcal{A}_{\left(-\infty, \tau_{j}\right)}^{\varepsilon}\right) \\
= & \mathbb{E}\left(f\left(\psi_{0-}^{T_{\delta, \varepsilon}}\right)\right) \mathbb{E}\left(g\left(\psi_{0+}^{T_{\delta, \varepsilon}}\right)\right)
\end{aligned}
$$

which complete the proof of the lemma.

Let us now conclude. For any bounded continuous function $f, g: \mathcal{B}(\mathbb{D}) \rightarrow \mathbb{R}$, let first $\delta \downarrow 0$, and then $\varepsilon \downarrow 0$ in the equality

$$
\mathbb{E}\left(f\left(\psi_{0-}^{T_{\delta, \varepsilon}}\right) g\left(\psi_{0+}^{T_{\delta, \varepsilon}}\right)\right)=\mathbb{E}\left(f\left(\psi_{0-}^{T_{\delta, \varepsilon}}\right)\right) \mathbb{E}\left(g\left(\psi_{0+}^{T_{\delta, \varepsilon}}\right)\right) .
$$

This yields

$$
\mathbb{E}\left(f\left(\psi_{0-}^{r_{0}}\right) g\left(\psi_{0+}^{r_{0}}\right)\right)=\mathbb{E}\left(f\left(\psi_{0-}^{r_{0}}\right)\right) \mathbb{E}\left(g\left(\psi_{0+}^{r_{0}}\right)\right),
$$

which ensures the independence of $\psi_{0+}^{r_{0}}$ and $\psi_{0-}^{r_{0}}$.

Moreover, $\left(u\left(r_{0}+x\right) ; x \geqslant 0\right)$ is $\sigma\left(\psi_{0+}^{r_{0}}\right)$-measurable, whereas $\left(u\left(r_{0}-x\right) ; x \geqslant 0\right)$ is $\sigma\left(\psi_{0-}^{r_{0}}\right)$-measurable. We obtain then, the second part of Theorem 2.

\section{The Cauchy case}

Assume that the initial potential $\psi_{0}$ is a Cauchy process. Bertoin ([6], Lemma 6 and Theorem 5) has proved that a.s. the range of $a$ is contained into the set of continuity points of $\psi_{0}$ and that $a$ is a.s. strictly increasing. In this section we will evaluate the size of the set of Lagrangian regular points.

THEOREM 3. - When the initial potential $\psi_{0}$ is a Cauchy process, the set $\mathcal{R}$ of Lagrangian regular points is a.s. uncountable and has Minkowsky dimension 0.

In particular, $\mathcal{R}$ has Hausdorff dimension 0.

Theorem 3 results from the following technical lemma.

Lemma 8. - Assume that the initial potential $\psi_{0}$ is a Cauchy process. Then for any $\delta>0$ there exists a finite constant $c(\delta)$ such that for any $0<\varepsilon<\delta / 2$ and $b \in \mathbb{R}$

$$
\mathbb{P}(\exists y=a(x) \in[0, \varepsilon] \text { with } x \in[b, b+\delta]) \leqslant c(\delta) . \varepsilon^{1-\delta} .
$$

The proof of this lemma is postponed to the end of the section.

Proof of Theorem 3. - One deduces from the strict monotonicity of $a$ that $\mathcal{R}$ is uncountable. Indeed, $a$ induces a bijection between the set of point of continuity of $a$ and $\mathcal{R}$.

Let us prove now that $\mathcal{R}$ has Minkowsky dimension 0. Again, the proof is closely connected to the proof of Theorem 4 in [6]. Our intention is to prove that for any $\delta>0$, 
$\mathcal{R}$ has Minkowsky dimension at most $2 \delta$. In this aim, we will split $[0,1]$ into small intervals of size $\varepsilon$, and prove that the number of intervals containing a regular point is at most $\varepsilon^{2 \delta}$.

For any fixed $n, \delta>0$ and $\varepsilon<\delta / 2$ define

$$
N_{\varepsilon}^{(n)}=\operatorname{card}\{k=0 \ldots[1 / \varepsilon] ; \exists a(x) \in[k \varepsilon,(k+1) \varepsilon], \text { with }|x| \leqslant n\} .
$$

One obtains with Fubini's theorem and the previous lemma the following upper bound

$$
\begin{aligned}
\mathbb{E}\left(\varepsilon^{2 \delta} N_{\varepsilon}^{(n)}\right) & =\sum_{k=0}^{[1 / \varepsilon]} \sum_{p=0}^{2 n / \delta} \varepsilon^{2 \delta} \mathbb{P}(\exists a(x) \in[k \varepsilon, k \varepsilon+\varepsilon] ; x \in[-n+p \delta,-n+p \delta+\delta]) \\
& \leqslant \sum_{k=0}^{[1 / \varepsilon]} \sum_{p=0}^{2 n / \delta} c(\delta) \cdot \varepsilon^{1+\delta} \leqslant \operatorname{cst}(n, \delta) . \varepsilon^{\delta} .
\end{aligned}
$$

Then take $\varepsilon=2^{-p}$. So

$$
\mathbb{E}\left(\sum_{p \geqslant 0} 2^{-2 p \delta} N_{2-p}^{(n)}\right)<\infty
$$

which ensures that a.s.

$$
\limsup _{p \rightarrow \infty} 2^{-2 p \delta} N_{2^{-p}}^{(n)}=0 .
$$

If we choose now $2^{-p}<\varepsilon \leqslant 2^{-p+1}$, the monotonicity of $N_{\varepsilon}^{(n)}$ in the variable $\varepsilon$ implies that

$$
\varepsilon^{2 \delta} N_{\varepsilon}^{(n)} \leqslant 2^{2 \delta} 2^{-2 p \delta} N_{2^{-p}}^{(n)}
$$

and in particular

$$
\limsup _{\varepsilon \downarrow 0} \varepsilon^{2 \delta} N_{\varepsilon}^{(n)}=0 .
$$

This exactly means that the Minkowsky dimension of $\{a(x) \in[0,1] ;|x| \leqslant n\}$ is at most $2 \delta$. We can choose $\delta$ and $n$ at our will, so the Minkowsky dimension of $\{a(x) \in$ $[0,1]\}$ is zero, and due to the stationarity of $u(x)$ the Minkowsky dimension of $\mathcal{R}$ is zero.

It only remains now to prove Lemma 8 .

Proof of Lemma 8. - Lemma 8 bears the same flavor as the result (2) in Section 2.1. The proof is then similar. In the proof of Theorem 1 we replaced a parabola by the graph of two $\beta$-stable subordinators, in order to deal with homogeneous Markov process. In the same way, we will replace here a parabola by an appropriated vertical cone. Let us explain this.

A Cauchy process $\psi_{0}$ is the sum of a symmetric Cauchy process $C$ and a drift: $\psi_{0}(y)=C(y)+d y$. Adding a drift to the potential $\psi_{0}$ (i.e. adding a constant to the initial velocity) has no effect on the set of regular points. In particular, we may assume that $d=0$. Remember that Bertoin [6] has proved that $C$ is continuous at any point of the range of $a$. We deduce in particular that for every regular point $r=a(x)$ 


$$
\begin{aligned}
C(r \pm h)-C(r) & \leqslant \frac{1}{2}(r \pm h-x)^{2}-\frac{1}{2}(r-x)^{2} \\
& \leqslant h(h / 2 \pm(r-x)) .
\end{aligned}
$$

Suppose that $r=a(x) \in[0, \varepsilon]$ with $x \in[b, b+\delta]$ and $h \in(0, \varepsilon+\delta)$. Then

$$
\left\{\begin{array}{l}
C(r+h)-C(r) \leqslant h((\varepsilon+\delta) / 2+\varepsilon-b), \\
C(r-h)-C(r) \leqslant h((\varepsilon+\delta) / 2+b+\delta) .
\end{array}\right.
$$

If we define

$$
Y^{-}(y)=C(y)+y\left(b+\frac{\varepsilon+3 \delta}{2}\right) \text { for } y \geqslant-\delta,
$$

then (9) implies that $Y^{-}$reaches a new maximum at $r$. Call now $\tau$ the first time after 0 where $Y^{-}$reaches a new maximum (notice that $0 \leqslant \tau \leqslant r \leqslant \varepsilon$ ). If $Y^{+}$denotes the process

$$
Y^{+}(y)=C(\tau+y)-C(\tau)+y\left(b-\frac{3 \varepsilon+\delta}{2}\right) \text { for } y \geqslant 0,
$$

then the first condition in (9) implies that $Y^{+}$does not reach a new maximum in $(\varepsilon, \varepsilon+\delta)$. Notice that $\tau$ is a stopping time for $C$, so $Y^{+}$and $Y^{-}$are two independent Cauchy process (with different drift). Putting the pieces together, one deduces that

$$
\mathbb{P}(\exists y=a(x) \in[0, \varepsilon] \quad \text { with } x \in[b, b+\delta]) \leqslant \mathbb{P}_{1} \mathbb{P}_{2},
$$

where

$$
\left\{\begin{array}{l}
\mathbb{P}_{1}=\mathbb{P}\left(Y^{-} \text {reaches a new max on }[0, \varepsilon]\right), \\
\mathbb{P}_{2}=\mathbb{P}\left(Y^{+} \text {reaches no new max on }[\varepsilon, \varepsilon+\delta]\right) .
\end{array}\right.
$$

Again, like in Lemma 7 of [6], we have

$$
\mathbb{P}_{2} \leqslant \operatorname{cst}(\delta) \times \phi(1 / \varepsilon)^{-1}
$$

where

$$
\phi(1 / \varepsilon)=\exp \int_{0}^{\infty} \frac{\mathrm{e}^{-s}-\mathrm{e}^{-s / \varepsilon}}{s} \mathbb{P}\left(Y^{+}(s) \geqslant 0\right) \mathrm{d} s .
$$

Using the scaling property of $Y^{+}$, one deduces that $\mathbb{P}\left(Y^{+}(s) \geqslant 0\right)$ does not depend on $s>0$ and equals

$$
\frac{1}{2}+\frac{1}{\pi} \arctan \left(b-\frac{3 \varepsilon+\delta}{2}\right)
$$

Thus, we have

$$
\mathbb{P}_{2} \leqslant \operatorname{cst}(\delta) \times \exp \left[\left(\frac{1}{2}+\frac{1}{\pi} \arctan \left(b-\frac{3 \varepsilon+\delta}{2}\right)\right)(\log \varepsilon)\right] .
$$


Once again, like in Lemma 7 of [6], we evaluate $\mathbb{P}_{1}$ by using time reversal. It is easily seen that then

$$
\begin{aligned}
\mathbb{P}_{1} & \leqslant \operatorname{cst}(\delta) \times \exp \left[\left(1-\mathbb{P}\left(Y_{s}^{-}\right)\right)(\log \varepsilon)\right] \\
& \leqslant \operatorname{cst}(\delta) \times \exp \left[\left(\frac{1}{2}-\frac{1}{\pi} \arctan \left(b+\frac{\varepsilon+3 \delta}{2}\right)\right)(\log \varepsilon)\right] .
\end{aligned}
$$

We finally deduce that

$$
\begin{aligned}
\mathbb{P}_{1} \mathbb{P}_{2} \leqslant & \operatorname{cst}(\delta) \\
& \times \exp \left[\left(1+\frac{1}{\pi} \arctan \left(b-\frac{3 \varepsilon+\delta}{2}\right)-\frac{1}{\pi} \arctan \left(b-\frac{\varepsilon+3 \delta}{2}\right)\right)(\log \varepsilon)\right] \\
\leqslant & \operatorname{cst}(\delta) \cdot \varepsilon^{1-2 \pi^{-1}(\delta+\varepsilon)} \leqslant \operatorname{cst}(\delta) \cdot \varepsilon^{1-\delta}
\end{aligned}
$$

in using the inequality $\arctan (x)-\arctan \left(x^{\prime}\right) \leqslant x-x^{\prime}$ for any $x^{\prime} \leqslant x$. Lemma 8 has been proved.

\section{Numerical illustration}

We give here a numerical illustration of the both cases studied in this paper. The simulation of $u$ has been made in breaking the line into 1600 points, and replacing a Lévy process by a random walk.

\section{1. $\alpha=0.85$}

The following simulation of $u$ (Fig. 3) has been made for $\alpha=0.85$ and $t=1$. One may notice a regular point around $x=260$.

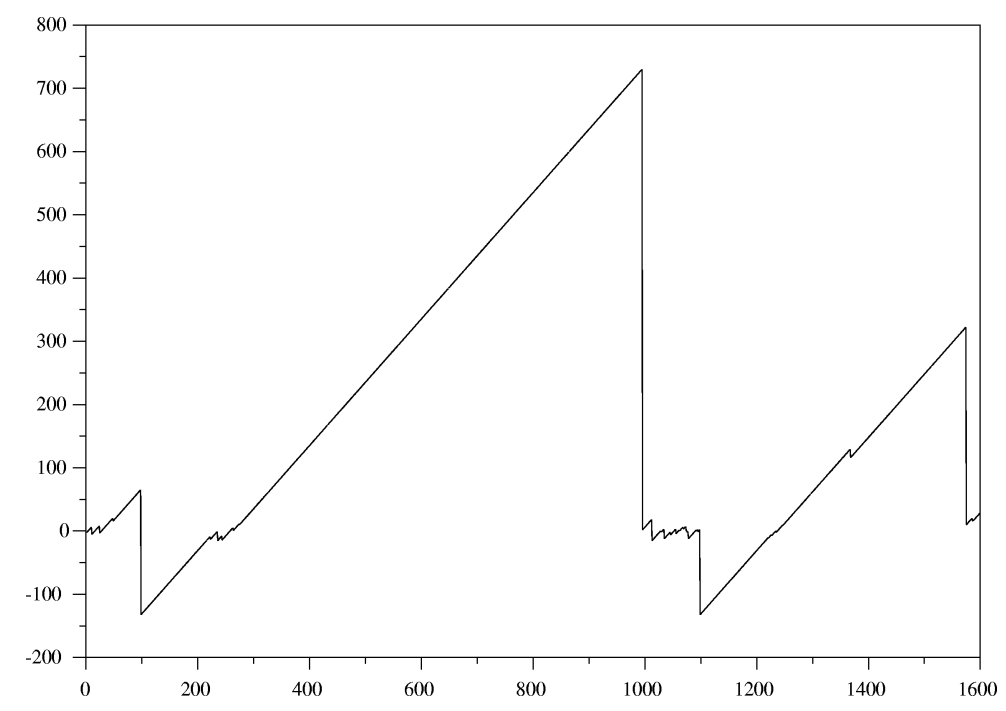

Fig. 3. 


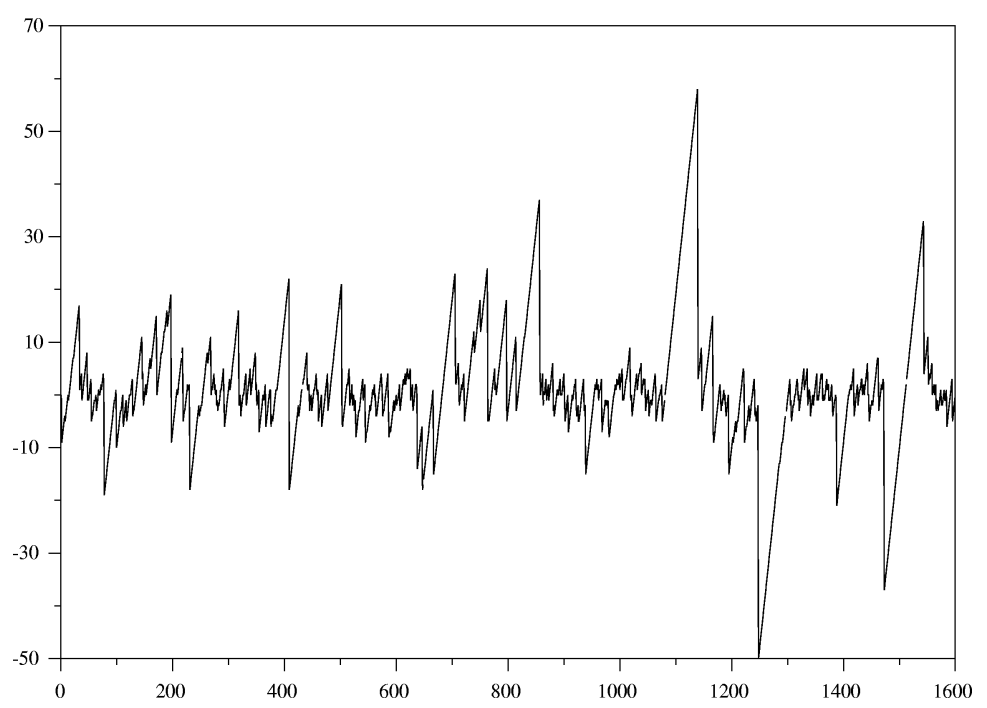

Fig. 4.

\section{2. $\alpha=1$}

We have here a simulation of $u$ (Fig. 4) in the Cauchy case at time $t=1$. One may notice the proliferation of small shocks.

\section{Acknowledgement}

I am very grateful to J. Bertoin for his guidance, without which this work would not have been possible.

\section{REFERENCES}

[1] Avellaneda M., E W., Statistical properties of shocks in Burgers turbulence, Comm. Math. Phys. 172 (1995) 13-38.

[2] Avellaneda M., Statistical properties of shocks in Burgers turbulence II, Comm. Math. Phys. 169 (1995) 45-59.

[3] Bertoin J., Lévy Processes, Cambridge University Press, Cambridge, 1996.

[4] Bertoin J., The inviscid Burgers equation with brownian initial velocity, Comm. Math. Phys. 193 (1998) 397-406.

[5] Bertoin J., Large deviation estimate in Burgers turbulence with stable noise initial data, J. Stat. Phys. 91 (1998) 655-667.

[6] Bertoin J., Structure of shocks in Burgers turbulence with stable noise initial data, Comm. Math. Phys. 203 (1999) 729-741.

[7] Burgers J.M., The Nonlinear Diffusion Equation, Dordrecht, Reidel, 1974.

[8] Cole J.D., On a quasi linear parabolic equation occuring in aerodynamics, Quart. Appl. Math. 9 (1951) 225-236.

[9] Frachebourg L., Martin Ph.A., Exact statistical properties of the Burgers equation, J. Fluid. Mech. 417 (2000) 69-99. 
[10] Fristedt B.E., Uniform local behavior of stable subordinators, Ann. Probab. 7 (1979) 10031013.

[11] Getoor R.K., Splitting times and shift functionals, Z. Wahrscheinlichkeitstheorie Verw. Gebiete 47 (1979) 69-81.

[12] Groeneboom P., Brownian motion with a parabolic drift Airy functions, Probab. Theory Related Fields 81 (1989) 79-109.

[13] Handa H., A remark on shocks in inviscid Burgers turbulence, in: Fitzmaurice et al. (Eds.), Non-linear Waves Weak Turbulence, Birkhäuser, Boston, 1992, pp. 339-345.

[14] Hawkes J., A lower Lipschitz condition for the stable subordinators, Z. Wahrscheinlichkeitstheorie Verw. Gebiete 17 (1971) 23-32.

[15] Hopf E., The partial differential equation $u_{t}+u u_{x}=\mu u_{x x}$, Comm. Pure Appl. Math. 3 (1950) 201-230.

[16] Janicki A., Woyczynski W.A., Hausdorff dimension of regular points in stochastic flows with Lévy $\alpha$-stable initial data, J. Stat. Phys. 86 (1997) 277-299.

[17] Leonenko N., Limit Theorems for Random Fields with Singular Spectrum, Math. Appl., Kluwers Academic, 1999.

[18] Ryan R., Large-deviation analysis of Burgers turbulence with white-noise initial data, Comm. Pure Appl. Math. 51 (1998) 47-75.

[19] She Z., Aurell E., Frisch U., The inviscid Burgers equation with initial data of Brownian type, Comm. Math. Phys. 148 (1992) 623-641.

[20] Sinai Y., Statistics of shocks in solutions of inviscid Burgers equation, Comm. Math. Phys. 148 (1992) 601-621.

[21] Woyczynski W.A., Burgers-KPZ Turbulence, Göttingen Lectures, Lectures Notes in Math., Vol. 1700, Springer, 1998. 